\title{
Alfonso Roque Albanese: Pionero de la Cirugía Cardíaca Latinoamericana. Homenaje de la Asociación Panamericana de Anatomía
}

\author{
Alfonso Roque Albanese: Latin American Pioneer of Heart Surgery. \\ Tribute from the Pan American Association of Anatomy
}

Ricardo J. Losardo ${ }^{1,5}$; Rolando Cruz Gutiérrez ${ }^{1,6}$; José C. Prates ${ }^{1,7}$; Alberto Rodríguez Torres ${ }^{1,8}$;
Nadir E. Valverde Barbato de Prates ${ }^{4,9}$; Manuel Arteaga Martínez ${ }^{2,10}$ \& Richard Halti Cabral $^{3,9}$

LOSARDO, R. J.; CRUZ, G. R.; PRATES, J. C.; RODRÍGUEZ, T. A.; PRATES, N. E. V. B.; ARTEAGA, M. M. \& CABRAL, R. H. Alfonso Roque Albanese: pionero de la cirugía cardíaca latinoamericana. Homenaje de la Asociación Panamericana de Anatomía. Int. J. Morphol., 35(3):1016-1025, 2017.

RESUMEN: Alfonso Roque Albanese (1906-2005) es considerado un pionero de la cirugía cardíaca Latinoamericana por sus importantes aportes. Fue miembro fundador de la Asociación Panamericana de Anatomía. Investigador y docente tanto de la anatomía como de la cirugía, uniendo ambas disciplinas.

PALABRAS CLAVE: Alfonso Roque Albanese; Asociación Panamericana de Anatomía; Anatomía; Cardiocirugía; Cirugía cardíaca; Latinoamérica.

\section{BIOGRAFÍA}

Alfonso Roque Albanese nació en Italia en 1906 y falleció en Argentina en 2005, a la edad de 99 años. Fue uno de los fundadores de la Asociación Panamericana de Anatomía. Además de ser una importante figura en esa disciplina, es considerado un pionero en cirugía cardíaca en Latinoamérica. Su primera gran pasión fue la anatomía, cuyo estudio nunca interrumpió. Estos conocimientos fueron siempre volcados a la cirugía, su segunda gran pasión. Sus actividades investigativas, docentes y asistenciales como médico siempre estuvieron presentes a lo largo de su vida (Fig. 1).

Los primeros tiempos. Alfonso Roque Albanese había nacido el 4 de mayo de 1906, en la ciudad de Corigliano, provincia de Cosenza, al sur de Italia. Su familia directa estaba formada por su madre (Rita), su padre (Cosme) y dos hermanos (Pascual y Serafina).

En esos años las posibilidades de progresar en Europa eran bastante escasas, razón ésta por la que su padre (labrador de escaso nivel de educación) probó suerte, como tantos otros, viniendo a la Argentina, país que por entonces se perfilaba como promisorio, permi-

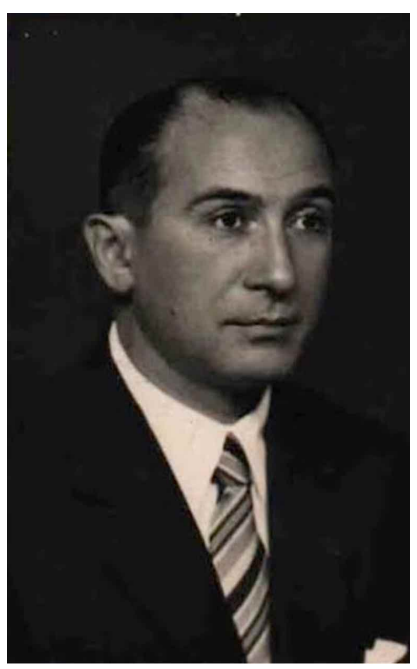

Fig. 1. Alfonso Roque Albanese (1906-2005).

\footnotetext{
${ }^{1}$ Presidente Honorario de la Asociación Panamericana de Anatomía (APA) y Co-Presidente de la Academia Panamericana de Anatomía (AcPA).

2 Presidente actual de la Asociación Panamericana de Anatomía (APA).

3 Secretario General actual de la Asociación Panamericana de Anatomía (APA).

${ }^{4}$ Ex Secretaria General de la Asociación Panamericana de Anatomía (APA) y Secretaria General actual de la Academia Panamericana de Anatomía (AcPA).

${ }^{5}$ Facultad de Medicina, Universidad del Salvador (USAL), Buenos Aires, Argentina.

${ }^{6}$ Facultad de Medicina, Universidad de Costa Rica (UCR), San José, Costa Rica.

7 Faculdade de Medicina, Universidade Federal de SÃo Paulo (UNIFESP), Sãn Pablo, Brasil.

${ }^{8}$ Facultad de Medicina, Universidad de Chile (UCh), Santiago, Chile.

9 Instituto de Ciencias Biomedicas (ICB), Universidade de SÃo Paulo (USP), São Pablo, Brasil.

${ }^{10}$ Facultad de Medicina, Universidad Nacional Autónoma de México (UNAM), Ciudad de México, México.
} 
tiendo "hacer la América", tal como se llamaba entonces a la posibilidad de mejorar sobre todo las condiciones económicas.

Es así que Cosme realizó dos viajes a la Argentina antes de hacer el tercero, que fue el definitivo. El resto de su familia, la madre y sus tres hijos, lo hizo al poco tiempo, a fines de 1910, embarcados en una de las naves más rápidas de esa época: el buque Princesa Mafalda, en el que también viajaría luego Carlos Gardel y otras personalidades de entonces.

Llegados al puerto de Buenos Aires pasaron su primera noche en el Hotel de los Inmigrantes, institución estatal y gratuita, que brindaba en forma transitoria alojamiento y comida a los extranjeros recién desembarcados.

Ya instalados, en su primer año, habitaron en una casa tipo "conventillo", donde cada familia vivía en una habitación con un baño y una cocina que se compartía entre varias familias. Este tipo de vivienda colectiva era alquilada frecuentemente por los inmigrantes que recién llegaban a la ciudad.

Tanto el padre como los dos hijos varones, trabajaron en el Mercado de Abasto (en la calle Corrientes), en la zona del barrio homónimo, donde también vivió el ya nombrado Carlos Gardel con su madre.

Así comienza esta aventura en busca de un mejor porvenir y sobre todo tentando la posibilidad de brindar a los hijos una formación que en Italia, por los problemas económicos existentes, era prácticamente imposible.

Alfonso Albanese hizo sus estudios: primario en la Escuela Martín Rodríguez y el secundario en el prestigioso Colegio Nacional Mariano Moreno; ambas instituciones públicas, de carácter gratuito, cercanas a su hogar. Por entonces Alfonso tocaba el violín en los cines mudos de la época, lo cual le proveía algún dinero extra para sus estudios y para la familia. Además, en sus ratos de diversión frecuentaba los bares ya que era un experto jugador de naipes y de billar, lo que en ocasiones le generó pérdidas monetarias y grandes disgustos. Así fue como una vez puso en juego el dinero para su inscripción en la Universidad (Régoli de Mullen, 2011).

La época universitaria. Llegó la época de los estudios universitarios y se decidió por la medicina. En 1924 preparó el ingreso a la Facultad junto a dos compañeros del secundario: Leoncio Fernández y Miguel Coronatto Paz. El primero, Leoncio, con el que compartiría una trayectoria hospitalaria en sus primeros años de médico y luego se destacaría como traumatólogo. El segundo, Miguel, quien no ingresaría a la universidad, pero con quien compartiría el desafío de la construcción de su Clínica y se destacaría por su actividad relacionada con el cine, la radio y la televisión.

Su hermano Pascual, algo mayor que él, primero se inclinó por los estudios filosóficos, pero luego se decidió también por la medicina, que inició cursando en la Universidad de La Plata, mientras que Alfonso lo hizo en la de Buenos Aires. Ambas universidades estatales y gratuitas. Alfonso se recibió en 1932, dos años antes que su hermano, quien lo hizo en 1934. Pascual, se especializó en Obstetricia y Ginecología, formándose en el Hospital Rivadavia. De esta manera, se estaba cumpliendo el sueño de los padres (Rita y Cosme) comenzando a hacerse realidad la formación de sus hijos en el país que los había cobijado y ofrecido tantas posibilidades.

Desde temprano, Alfonso, mostró un interés especial y un gran deslumbramiento por la Anatomía. Esto motivó a su docente de primer año, Atilio Costa, para incorporarlo a la Cátedra de Anatomía Descriptiva (a cargo de Joaquín López Figueroa) en calidad de Ayudante. En segundo año cursó Anatomía Topográfica con Avelino Gutiérrez, quien se jubiló en 1927, sucediéndolo Eugenio Antonio Galli, de una gran dedicación a la anatomía del corazón. Alfonso Albanese también cursó Fisiología con el entonces joven Bernardo Houssay, quien había fundado en la Facultad el "Instituto de Fisiología", verdadero semillero de profesionales en esa disciplina. En 1927, Albanese se incorporó como Ayudante a la Cátedra de Anatomía Topográfica. Durante dos años se distrajo de sus estudios y se dedicó a participar en política universitaria, actividad que posteriormente abandonó. Antes de recibirse fue Practicante en los Hospitales Durand y Piñero, sucesivamente (Albanese, 1961; Lucas, 1995; Martí, 2005; Régoli de Mullen).

Tal vez, la zona de Italia de dónde provenía signaba como premonición el futuro del doctor Albanese, ya que allí se practicaron las primeras disecciones anatómicas de las que se tienen noticias (Losardo et al., 2016).

La familia. En mayo de 1933, Alfonso contrajo matrimonio con Nélida Bardi, a quien conocía desde años atrás. Ella será su compañera para toda la vida. Tres meses después falleció la madre de Albanese, Rita. Nélida quedó embarazada y nació el primer hijo: Alfonso Miguel, en 1934, y cuatro años después, en 1938, el segundo hijo del matrimonio: Eduardo Francisco. La familia Albanese vivió entonces, en la casa de los padres de Nélida, quienes tenían allí un comercio dedicado a la venta de telas y ropa. En el primer piso de esa propiedad, vivió el matrimonio Albanese con sus dos hijos durante varios años (Régoli de Mullen). 
El Hospital Rawson. Ahora había llegado el momento de elegir un hospital para perfeccionarse y adquirir experiencia. En esa época se ingresaba al hospital sin recibir ninguna retribución económica y el sustento se conseguía a través de la consulta privada.

Primero, Albanese pensó en especializarse con Pedro Chutro, profesor de Cirugía, quien lo había impresionado desde la época de estudiante. Chutro era Jefe de Sala en el Hospital San Roque (actual Ramos Mejía). Este destacado maestro tenía un extenso currículum con distinciones internacionales. En los años 20 ya era una personalidad de renombre como cirujano, dentro y fuera del país. Desgraciadamente se enfermó y falleció en la década del 30, a los 57 años.

Entonces apareció en la vida de Albanese otro maestro: Ricardo Finochietto. Como a Chutro lo había conocido en su época de estudiante. Finochietto le había tomado examen de técnica quirúrgica. En este momento, años 30, Finochietto era Jefe de Clínica (cargo equivalente a subjefe) en el Hospital Rawson, del cual su hermano Enrique era el Jefe de Sala o Servicio (en el Pabellón 9). Ese era un hospital muy especial pues ostentaba un alto nivel profesional. Enrique Finochietto era considerado uno de los talentos mundiales de la cirugía. Junto con Chutro eran los dos cirujanos argentinos más reconocidos internacionalmente (Figueroa, 1993; Lucas, 1995; Régoli de Mullen; Buroni, 2016).

Para ubicarnos en la época, señalemos que los primeros grandes cirujanos argentinos: Ignacio Pirovano y Alejandro Posadas inspiraron las "escuelas quirúrgicas" que se desarrollaron entonces en Buenos Aires, donde se destacaron las de Arce, Chutro, Roccatagliata y Finochietto. Esta última dio nacimiento a una pléyade de discípulos sólo comparable con los que formó Pirovano (Guerrino, 1981; Vacarezza, 1981; Figueroa; Gotta \& Buzzi, 2012).

En 1931, Ricardo Finochietto fue nombrado Jefe de Sala en el Hospital Alvear donde permaneció dos años. Luego volvió al Hospital Rawson, pero ya como Jefe de Sala y formó su propio Servicio de Cirugía (en el Pabellón 2). Alfonso Albanese conocía como trabajaba ese equipo. Los doctores Finochietto tenían un altísimo nivel de exigencia. En agosto de 1932, Albanese, con seis meses de graduado, le manifiesta el deseo de integrarse a su Servicio. Luego de presentar su Tesis de anatomía de cuello (como requisito) fue aceptado, primero en forma provisoria por dos semanas y luego de manera definitiva. A partir de allí quedó incorporado al Servicio, en 1933, en calidad de Médico Asistente; y en 1938, como Médico Agregado. Fue integrante del primer grupo de discípulos de esta Escuela (Albanese, 1961; Lucas,
2005; Martí; Régoli de Mullen).

En la Escuela de Finochietto se ponía especial énfasis en la "técnica quirúrgica" (basada en el conocimiento de la Anatomía Quirúrgica) pero no se descuidaba, en el preoperatorio, la "táctica quirúrgica" (basada en la limitada fisiopatología y anatomía patológica que se conocía entonces). También se hacía cirugía experimental en perros a manera de ejercitación antes de hacer cirugía en humanos. Los hermanos Finochietto daban especial valor a la Anatomía, esto Ricardo lo llevaba en su alma. Eran cirujanos con gran conocimiento anatómico, por ello veían al joven Alfonso de una forma distinta a la del resto de sus discípulos (Finochietto, 1962; Pataro, 1983; Figueroa; Zancolli, 2002).

La relación que comenzó a forjarse entre $\mathrm{R}$. Finochietto y Albanese (se llevaban 20 años de diferencia de edad) se cimentó, sobre todo, en el conocimiento de la anatomía y en el permanente interés de Alfonso por colaborar en todo cuanto Ricardo proponía. Se demostraron mutuamente gestos y actitudes especiales de estima profesional y respeto humano.

Todo empezó con una frenicectomía (o frénicoexéresis) en un paciente con tuberculosis pulmonar, como tratamiento paliativo. La idea era conseguir una técnica que utilizara una incisión pequeña, para que la cicatriz pasara inadvertida, ya que esta enfermedad en aquella época era un estigma. Albanese propuso una técnica que fue aceptada y reemplazó a la que había ideado Ricardo Finochietto con anterioridad. Entre 1935 y 1937, Finochietto llevó a Albanese a distintos hospitales para que operara a pacientes tuberculosos, en Buenos Aires y en Córdoba. Las sierras cordobesas eran conocidas por su clima favorable para estos enfermos y allí se creó la "Escuela Tisiológica Argentina". De esta manera, Albanese se hizo muy conocido entre sus colegas (Lucas, 1995; Régoli de Mullen).

En 1936, R. Finochietto invitó al padre de Alfonso, Cosme, para que observara una cirugía que practicaba su hijo en el hospital. Así se cumplió el sueño que coronó tantos esfuerzos y anhelos puestos en la formación de sus hijos. A pesar de la escasa educación que traían de su Italia natal y gracias a la gran movilidad social que existía en la Argentina, se hizo posible con un gran esfuerzo el famoso dicho de "mi hijo el doctor" (Saadia, 2009; Régoli de Mullen).

Clínicas Albanese. La primera Clínica que tuvo Albanese fue en 1940. Era pequeña y estaba ubicada en las calles Alsina y Combate de los Pozos, en la ciudad de Buenos Aires. Fue el resultado del esfuerzo junto con un compañero de hospital: Santos Luchetti. La institución llevó el nombre de am- 
bos y allí, en 1941, Albanese practicó su primera cirugía cardíaca: un ducto arterioso persistente, en una niña, con un resultado exitoso.

Luego, Alfonso Albanese se quedó solo en la Clínica e invitó a diez compañeros del hospital (todos discípulos de R. Finochietto) a formar parte de la misma. En ese momento la Clínica pasó a llamarse "Sanatorio Central de Cirugía". El proyecto de "trabajo especializado" que propuso no prosperó y Alfonso decidió retirarse del emprendimiento.

Después siguió un proyecto mayor e individual: un centro médico de avanzada sobre el modelo de la Mayo Clinic de Estados Unidos de América. Así es que compró una antigua casa y construyó un edificio que constaba de subsuelo, planta baja y cuatro pisos, en la calle Bartolomé Mitre esquina Larrea, en la ciudad de Buenos Aires. Para este desarrollo contó con la colaboración de tres amigos del colegio secundario, que ayudaron a llevarlo adelante (entre ellos, el citado Coronatto Paz). La energía que le insumía este proyecto hizo que debiera renunciar al Hospital Rawson en 1946. Inauguró la nueva Clínica a mediados de 1948 (esta construcción actualmente forma parte del "Sanatorio Mitre"). Un detalle: en el último piso vivió con su familia, dejando la casa de sus suegros (Lucas, 2005; Régoli de Mullen) (Fig. 2).

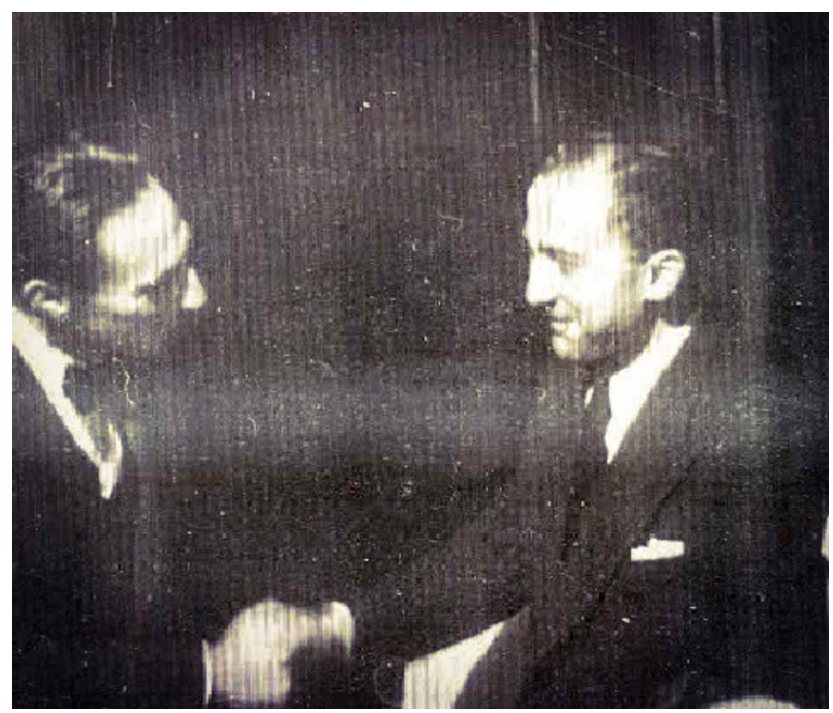

Fig. 2. Ricardo Finochietto (derecha) felicitando a Alfonso Roque Albanese por una operación de anastomosis porto-cava.

La Clínica contaba con laboratorio y radiología. Su tecnología estaba siempre actualizada y resultaba de avanzada para la época (cateterismos, angiografías, aortografías, flebografías, etcétera). Tenía morgue y biblioteca, esta última muy completa y actualizada. Además, la Clínica tenía un área de cirugía experimental, con una actividad continua y muy intensa. Lo acompañaron los doctores Rascovsky,
Javkin y Saadia, quienes en la década del 60 formaron parte del equipo con el que Albanese realizó las cirugías cardíacas extracorpóreas. Durante los 15 años que funcionó la Clínica, Albanese perfeccionó y creó diversas técnicas quirúrgicas basadas en su dominio de la anatomía. Estas prácticas eran observadas por becarios del interior del país y de distintos países latinoamericanos, a quienes Albanese ayudaba económicamente en su estadía. Albanese tenía una capacidad extraordinaria de trabajo y las jornadas quirúrgicas llegaban a las 15 horas diarias, donde se sucedían operaciones con distintas patologías. Si bien la Clínica era particular o privada, también se atendían y operaban pacientes de bajos recursos económicos en forma gratuita. Ambos hechos ponen de manifiesto su generosidad y bonhomía (Labra, 1956; Rascovsky, 2002; Lucas, 2005; Saadia).

El Servicio de Patología de la Clínica estaba a cargo de Moisés Polak, uno de los mejores en ese momento, lo acompañaba el joven Rolbider Feola. Los cardiólogos eran los destacados Roberto Vedoya y José González Videla, incorporándose luego el joven Carlos Bertolasi (Régoli de Mullen).

René Leriche, en 1950, visitó la institución y en 1951 lo hizo Helen B. Taussig. El primero, había iniciado en el mundo la cirugía del simpático periférico y su efecto sobre las arterias. La segunda, fundó a nivel mundial la Cardiología Pediátrica, y produjo un cambio radical en la mentalidad de los cardiólogos y de los pediatras frente a las cardiopatías congénitas e ideó la primera cirugía para la Tetralogía de Fallot (Christmann et al., 1975; Régoli de Mullen; Cruz Gutiérrez \& Cruz Cruz, 2012).

Albanese operó el primer caso de "bebes azules" (Tetralogía de Fallot) en 1947 y constituyó el primero operado con éxito en Sudamérica. En su Clínica se le realizó a Taussig un homenaje con la presencia de más de 30 niños que habían sido operados exitosamente por él (Lucas, 2005; Régoli de Mullen).

La Clínica Albanese tuvo su esplendor entre 1948 y 1959, período que podemos señalar como "primera época", donde se crearon técnicas, se desarrollaron estudios y se llevaron a cabo operaciones que eran pioneras en su tipo. Durante 1959 y 1963, "segunda época", Albanese retomó la actividad hospitalaria desarrollándola en forma simultánea con la de la Clínica. También en este período se recibieron de médicos sus dos hijos, ambos con diploma de honor, quienes colaboraron con él en las cirugías, la investigación y la docencia.

Albanese dejó este emprendimiento en 1963, un año después del fallecimiento de su querido y gran maestro Ricardo Finochietto, todo esto le causó una gran depresión, de 
la cual se sobrepuso con el apoyo de su esposa y sus dos hijos médicos.

Hospital Fiorito. Después de 13 años vuelve a la labor hospitalaria. En 1959, ingresa al Hospital Fiorito (en la zona sur del Gran Buenos Aires), como Jefe del Servicio de Cirugía General. En esta oportunidad se le tributó un merecido homenaje en la casa de Edison Otero, fundador y Jefe de Cardiología de ese hospital. La presencia de Albanese en el Fiorito, 1959-1962, marcó un antes y un después en la historia de la cirugía en ese lugar. Replicó su querida Escuela del Hospital Rawson en todos sus aspectos. Hoy en la Sala de Cirugía de ese hospital, hay una placa que recuerda su paso por allí y la dedicación, cariño y esmero que puso en su labor (Rascovsky; Saadia; Régoli de Mullen).

Actividad societaria. Albanese desarrolló una intensa actividad en distintas asociaciones dedicadas a la docencia e investigación médica.

En el campo de la anatomía, en la Asociación Panamericana de Anatomía (de la que fue "Miembro Fundador", 1966) y en la Sociedad-Asociación Rioplatense de Anatomía, donde recibió altos honores y distinciones por sus importantes contribuciones (entre ellas, la de "Miembro Emérito", 1998). Participó de los Congresos Panamericanos de Anatomía en México (1966), Caracas (1969), Nueva Orleans (1972), San Pablo (1978), Buenos Aires (1981) y Punta del Este (1984). También fue miembro de la Sociedad Chilena de Anatomía Normal y Patológica, que se convertiría años después en la Sociedad Chilena de Anatomía; y de la American Association of Anatomists. En el Primer Congreso de Anatomistas del Cono Sur, realizado por la Asociación Rioplatense (argentino-uruguaya) y la Sociedad Chilena de Anatomía, en la ciudad de Mendoza, en 1999, se instauró el premio anual "Alfonso Roque Albanese" al mejor trabajo realizado por estudiantes con el fin de estimular la investigación anatómica desde los inicios de la formación, en reconocimiento al concepto que tenía sobre la enseñanza integral de la medicina (Albanese, 1961; Martí; Losardo, 2009; Losardo et al., 2015).

Fue miembro de diversas sociedades nacionales de: cancerología, cardio-angiología, cirugía, endocrinología, gastroenterología, entre otras. Fue presidente de las Argentinas de Cirugía Torácica (1951) y de Angiología (1952), de las que asimismo fue miembro fundador. Esta última hoy se denomina Asociación Argentina de Angiología y Cirugía Cardiovascular. Obtuvo altas nominaciones como la de Miembro Honorario Nacional de la Asociación Médica Argentina (AMA), que también lo distinguió como Maestro de la Medicina. La Fundación Escuela Quirúrgica Finochietto (que funciona en la AMA) lo designó Miembro de Honor (2004).
A nivel internacional fue el primer Presidente del Capítulo Sudamericano de la International Angiology and Cardiovascular Society (1952) que dio origen en poco tiempo a la fundación de las Sociedades Nacionales en Argentina, Brasil, Chile, Uruguay y Venezuela. Fue distinguido como Presidente Honorario de dicho Capitulo (1954). También fue "Miembro Honorario" del American College of Angiology (de Estados Unidos de América), de la Sociedad Mexicana de Angiología, de la Sociedade Brasileira de Angiologia, de la Sociedade Brasileira de Cirurgia Cardiaca, Sociedade Medica São Lucas, de San Pablo y de la Sociedad Chilena de Cirugía. A nivel internacional, en 1986, fue distinguido con la "Orden al mérito angiológico René Leriche, Maestro de la cirugía cardiovascular". Además, se desempeñó como miembro del comité editorial de las revistas Angiology y Journal of Vascular Disease, de Estados Unidos de América (Albanese, 1961; Lucas, 1995; Lucas, 2005; Martí; Régoli de Mullen).

Actividad docente. Esta labor la inició en 1926, siendo aún alumno, desempeñándose como Ayudante ad honorem en la Cátedra de Anatomía Descriptiva en la Universidad de Buenos Aires (UBA). En 1929 fue Ayudante de Trabajos Prácticos y en 1943 fue nombrado Profesor Adjunto. En 1957 fue Profesor Titular Interino. En 1961 se retiró de la docencia retornando a la misma en 1969 en calidad de Asesor en Docencia y Disección en la Escuela de Ayudantes de la Tercera Cátedra de Anatomía. En 1974 fue designado Profesor Consulto y en 1980 Profesor Emérito (Albanese, 1961; Martí).

En la Universidad del Salvador (USAL) fue profesor de la Cátedra de Anatomía desde 1966 y colaboró con Luis Dellepiane, que estaba a cargo de ella desde su creación. En esta institución ejerció la docencia de pre y postgrado. Sus dos hijos también fueron profesores de esta casa de altos estudios. En sus últimos 35 años de vida mostró una gran dedicación a esta tarea y en el Museo de la USAL se encuentran preparados disecados por él. En 1974 lo nombraron Profesor Consulto y en 1980, Maestro. Hasta sus últimos días dictó cátedra para graduados, ayudantesdocentes y alumnos. A sus 99 años siguió transmitiendo su conocimiento con entusiasmo y alto nivel profesional. $\mathrm{Su}$ última clase la dictó el 18 de julio. El 11 de agosto de 2005 falleció, en Buenos Aires, de una estenosis aórtica complicada (Martí; Régoli de Mullen).

En el Colegio Argentino de Cirugía Cardiovascular, que depende de la Asociación Argentina de Angiología y Cirugía Cardiovascular, dictó clases de anatomía quirúrgica para el Curso de Posgrado. Por su labor docente y su trayectoria, las autoridades de este Colegio, lo nombraron Maestro de la Cirugía Cardiovascular Argentina (2003). Es de 
destacar que esta institución, cuenta con dos aulas principales, una lleva su nombre y la otra, el de René Favaloro, máximos representantes nacionales de la cirugía cardíaca en dos épocas sucesivas (Lucas, 2005; Martí; Saadia).

Pionero de la cirugía cardiovascular. Hacia fines de los años 30 se estaban recién iniciando las especialidades médicas. Los hermanos Finochietto impulsaban enfáticamente el sentido de la especialización entre sus discípulos y tuvieron una influencia decisiva en ellos. Albanese no fue ajeno a esta situación y junto con Vicente Pataro (su ayudante), en 1935, comenzaron a orientarse hacia la Cirugía Vascular y la Angiología. En ese entonces riesgosa y con alto índice de morbi-mortalidad.

Fue muy importante en la carrera de Alfonso Roque Albanese el conocimiento detallado y práctico que poseía de la anatomía del corazón y del tórax. Esto se vio reforzado con sus disecciones que ilustraron el libro "Corazón. Estudio descriptivo y topográfico" de su profesor de anatomía Eugenio Galli, quien también enseñaba en la Universidad de La Plata. En esta obra colaboró otro joven anatomista y disector de esta última casa de estudios, José María Mainetti, amigo de Alfonso, que luego se destacaría como cirujano oncólogo (Lucas, 1995; Saadia).

En 1941, con menos de 10 años de recibido, realizó su primera cirugía cardíaca. En esta especialidad fue un pionero (recordemos que el mundo de la cirugía cardíaca se inició en 1938 con Robert Gross), Albanese fue el tercero en Argentina en operar un paciente con un ducto arterioso persistente (el 19 de diciembre de 1941), el primero había sido Enrique Finochietto y el segundo Oscar Cames (Albanese, 1961; Lucas, 2005; Saadia; Régoli de Mullen; Cruz Gutiérrez \& Cruz Cruz).

A partir de allí, el doctor Albanese sumaría otros casos de ducto arterioso e iniciaría los casos de Tetralogía de Fallot (la primera, el 1 de abril de 1947) y de coartación de aorta (la primera, el 1 de agosto de 1947). Señalemos que fue en 1944 cuando se iniciaron estas dos cirugías en el mundo, con Alfred Blalock y Clarence Crafoord, respectivamente. Entre 1946 y 1950 publicó sus experiencias en el Día Médico, La Semana Médica, La Prensa Médica Argentina, etcétera, que registran su intensa actividad. También presentó sus casos operados en las sesiones de la Sociedad Argentina de Cirugía Torácica donde los comentarios finales estuvieron a cargo del prestigioso Jorge Alberto Taiana (Albanese, 1961; Martí; Saadia; Régoli de Mullen; Cruz Gutiérrez \& Cruz Cruz).

Tanto la cirugía del ducto arterioso como la de coartación de aorta eran operaciones "curativas" pero la de la
Tetralogía de Fallot era "paliativa”. Las tres se hacían "a corazón cerrado". En la última, habría que esperar el desarrollo tecnológico necesario (máquina de circulación extracorpórea) que permitiera realizar la cirugía "a corazón abierto" (detenido y vacío) para lograr la curación definitiva. Esto se lograría a mediados de la década de 1950 (Christmann et al.; Lucas, 2005).

Para ubicarnos en la época, destaquemos algunos hechos. El primero que, recién en 1940, se había terminado de conocer el sistema $\mathrm{Rh}$, que permitía realizar las trasfusiones de sangre evitando las reacciones de incompatibilidad, procedimiento que acompañaba a estas cirugías. El segundo que en 1941 se descubrió la heparina, y ello marcó un antes y un después en la cirugía cardiovascular. En la era pre-heparínica se operaba contra-reloj y las complicaciones trombóticas eran muy frecuentes. Finalmente, el tercero que tanto los estudios de diagnóstico para las enfermedades cardíacas como la anestesia general eran básicas y primitivas. En este contexto es que se inició y dio sus primeros pasos la cirugía cardíaca en el mundo.

En 1948, Albanese ideó un instrumento (valvulótomo) para el tratamiento de la estenosis valvar atrio-ventricular izquierda (mitral) "a corazón cerrado". Desarrolló esta técnica propia en más de 100 casos y fue presentado en las sesiones de la Sociedad Argentina de Cirugía Torácica y en el IV Congreso Latinoamericano de Cardiología (Albanese, 1961; Lucas, 1995; Martí).

En agosto de 1950, en oportunidad del Congreso conjunto: el Argentino de Cirugía y el del Internacional College of Surgeons, Albanese realizó la primera operación cardíaca transmitida por circuito cerrado de televisión, realizada en el país. Los comentarios estuvieron a cargo de Ricardo Finochietto y fue practicada en el Hospital Rivadavia, siendo seguida por espectadores en la Facultad de Medicina de la UBA y desde el Hotel Alvear. Al año siguiente, se iniciaba la transmisión de televisión comercial y con programas regulares en Argentina. Ambos proyectos fueron posibles gracias al singular y multifacético, Enrique Telémaco Susini, médico otorrinolaringólogo de fama internacional (Albanese, 1961; Lucas, 1995; Martí; Régoli de Mullen; Devoto, 2016).

En diciembre de 1951 dictó -en su Clínica y en la Facultad de Medicina de la UBA- el primer curso latinoamericano de cirugía cardíaca y vascular al que concurrieron cirujanos argentinos y del resto del continente, los que vueltos a sus países fueron pioneros en cirugía cardíaca (Albanese, 1961; Lucas, 2005; Martí; Saadia; Régoli de Mullen). 
En 1951 llegó a tener una casuística muy importante que lo ubicaba en el quinto lugar en el mundo en cirugía cardíaca. Un año después duplicó sus casos de cardiopatías congénitas tal como lo registran sus publicaciones. En 1955, ocupaba el cuarto lugar mundial, detrás de dos equipos norteamericanos y uno sueco (demostrado en el congreso internacional en Detroit, Estados Unidos de América). Todas esas experiencias simultáneamente fueron presentadas en distintas asociaciones y congresos médicos. En 1957 publicó en el Día Médico: "Cirugía de las Cardiopatías Congénitas (363 operados)", un análisis de 15 años de experiencia en clínica y tratamiento.

Entre 1941 y 1962 había realizado en su clínica 583 operaciones cardíacas: Tetralogías de Fallot, ductos arteriosos, coartaciones de aorta, estenosis e insuficiencias de la valva atrio-ventricular izquierda, pulmonares y aórticas, comunicaciones interatriales, trasposiciones de grandes vasos, pericarditis constrictivas y exudativas, entre otras. Así contribuyó con los primeros casos de cirugía cardíaca publicados en el mundo. También tuvo una casuística numerosa en operaciones de cáncer de mama, estómago, colon y recto, útero y ovarios, etcétera; así como cirugías de las paredes torácicas (tórax excavado) y abdominal (hernias y eventraciones) (Martí; Saadia; Régoli de Mullen) (Figs. 3, 4 y 5).

Contribuciones originales en cirugía. Perfeccionó e ideó métodos novedosos para operaciones de distintos órganos, que fueron publicados en libros y en revistas médicas.

Entre sus libros mencionamos: el de "Pancreatitis aguda", de 1942. Luego, el "Manual de bloqueos anestésicos del sistema neurovegetativo: Anestesias del Simpáti-

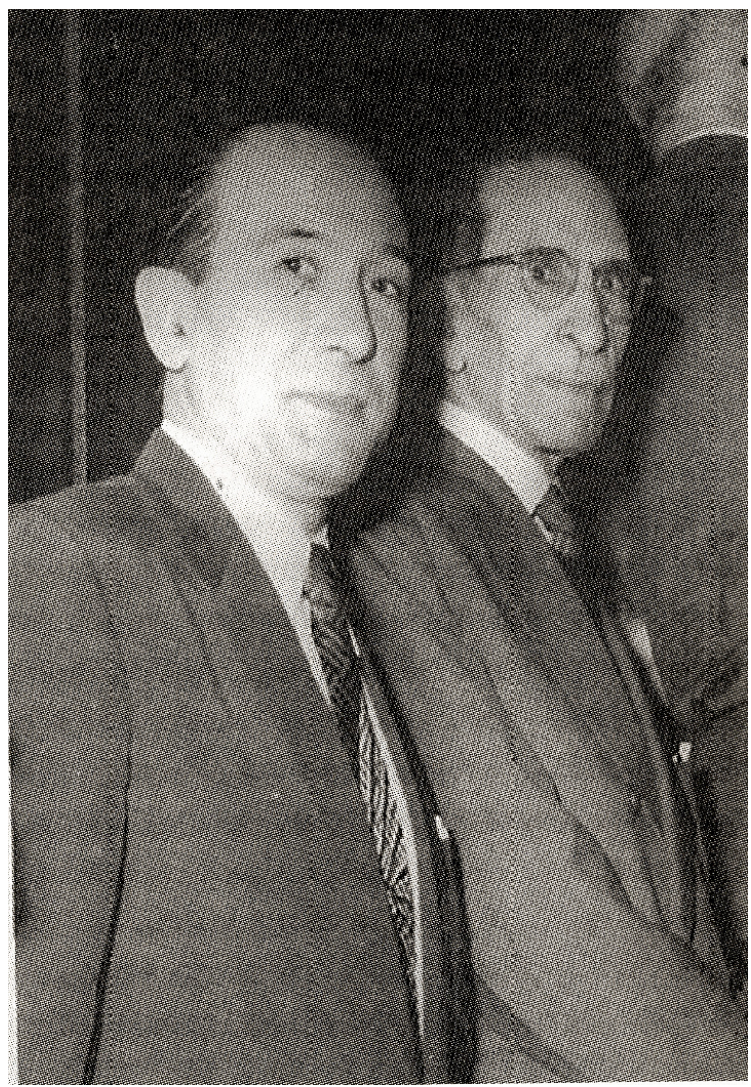

Fig. 3. Alfonso Roque Albanese (izquierda) con Eugenio Galli en la Facultad de Medicina (UBA)

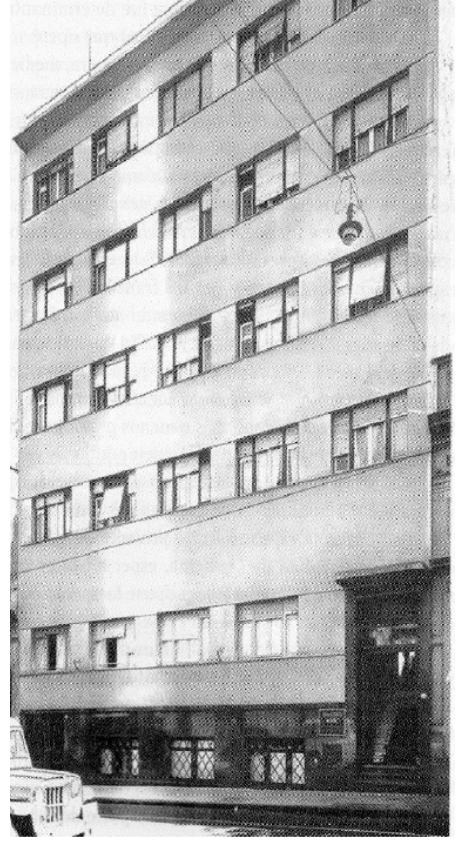

Fig. 4. Clínica Albanese (en la calle Bartolomé Mitre) co", de 1947, prologado por Ricardo Finochietto y que en los años 50 se constituyó en lectura de cabecera en los países de habla hispana. También en 1947 el libro "Cirugía de la hipertensión arterial", editado con aval de Ricardo Finochietto. Estos tres primeros libros, tratan sobre la anatomía quirúrgica del simpático y su aplicación terapéutica. Fue un pionero en cirugía del sistema simpático. Algunos años más tarde, en 1977, "El apéndice, la apendicitis y la apendicectomía", libro extraordinario que aún acompaña a muchos profesionales jóvenes. Una mención espe-

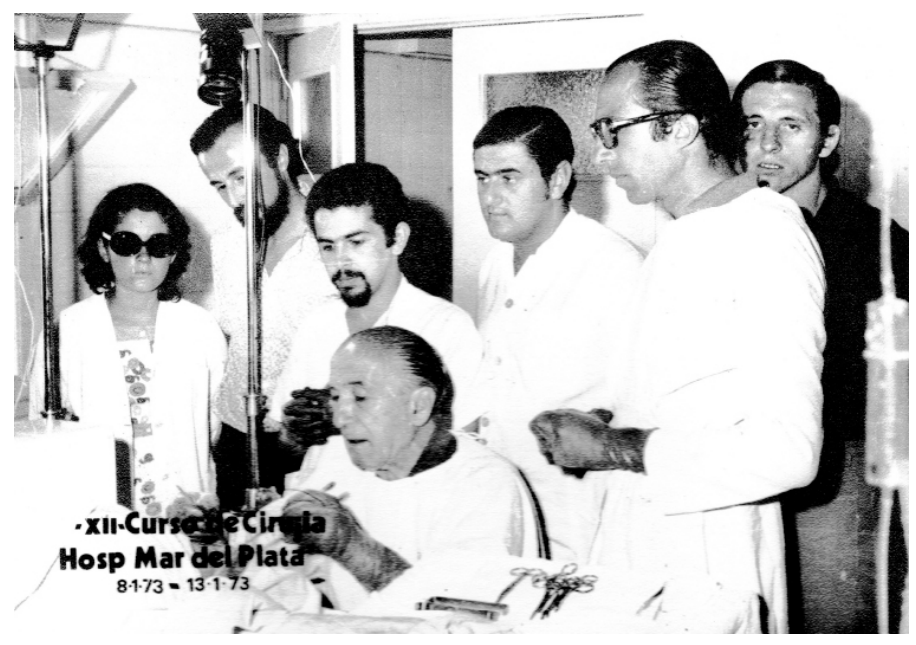

Fig. 5. Alfonso Roque Albanese (sentado) en un Curso de Posgrado en Mar del Plata, acompañado por su hijo Eduardo (de lentes). Detrás del maestro, un joven cirujano y anatomista, Jorge Moscol Gonzáles. 
cial merece su tesis doctoral “Anatomía del cuello", de 1932, que le abrió las puertas a la Escuela del Hospital Rawson a través de Ricardo Finochietto y donde se manifiesta su temprano entusiasmo en relacionar las disciplinas básicas (anatomía, embriología e histología) con la cirugía.

Larga es la lista de todas las investigaciones y aportes del Dr. Albanese en diferentes temas como cirugía de las glándulas tiroides y paratiroides, cirugía del nervio frénico, cirugía del simpático, cirugía del cáncer de mama, cirugía linfática, cirugía gastrointestinal, cirugía esplénica, cirugía de la vesícula, cirugía de várices de miembros inferiores, cirugía de la vena cava inferior, cirugía de la hipertensión portal, cirugía de las eventraciones abdominales, etcétera (Albanese, 1961; Albanese, 1977; Finochietto, 1962; Martí; Saadia; Régoli de Mullen).

En cirugía oncológica fue uno de los pioneros a nivel mundial en proponer una conducta quirúrgica menos agresiva en cáncer de mama. Describió una técnica original (1942), más conservadora, que reducía la extensión de los tejidos resecados y disminuía la morbilidad en estas pacientes (Albanese, 1961; Bernardello et al., 1984; Lucas, 1995).

Fue el creador del método de la biopsia cérvicomediastínica (1942) que publicara en la Argentina antes que Albert Daniels (1949) lo hiciera en Estados Unidos de América. La técnica de este último estaba restringida a nivel cervical (Albanese, 1961; Martí; Régoli de Mullen).

Su método para el tratamiento de grandes eventraciones o evisceraciones de la pared abdominal, que él denominó "incisiones de descarga" (1946), consiste en relajar la zona de las suturas mediante incisiones a distancia, fue reconocido mundialmente como un método original y un valioso aporte a la cirugía de la pared abdominal y que continúa utilizándose (Albanese, 1961; Apeceche, 1980; Majul, 1981; Pastorino, 1982; Cano et al., 2016).

En el tratamiento de la hipertensión portal por cirrosis hepática realizó en su Clínica, en 1949, la primera operación en Latinoamérica de anastomosis porto-cava latero-lateral, previniendo las graves hemorragias por ruptura de las várices esofágicas (Albanese, 1961; Martí; Régoli de Mullen).

Su pasión fue investigar las migraciones que hacían los órganos y sus estructuras desde el feto hasta su ubicación definitiva en el adulto; y con ello las fascias de coalescencias y las láminas vasculares en todo el cuerpo, desde el cuello al perineo. Su "lámina duodeno-retromesentérico-cólica derecha" (1953), mantuvo su atención durante casi toda su vida. Su última publicación la realizó a los 91 años en La Prensa Médica Argentina (1997). Su co- nocimiento permitía en la cirugía abdominal utilizar los planos de clivaje adecuados y movilizar los órganos gastrointestinales con el menor trauma posible, evitando tracciones y torsiones (Albanese, 1961; Albanese, 1977; Finochietto; Brana et al., 1983).

Legado. Albanese unió la Anatomía a la Cirugía, hecho que, si bien no era nuevo, su enfoque era diferente.

Sostenía que la enseñanza de la Anatomía era fundamentalmente disección cadavérica, caso contrario quedaba restringida y limitada en su expresión.

A la Anatomía la interpretaba según su fin, que para él era la Cirugía, herramienta fundamental en aquella época del tratamiento médico. La anatomía quedaba así por encima de la cirugía, vale decir que la cirugía se adaptaba a la anatomía, lo cual fue toda una novedad. Relacionaba anatomía, embriología e histología con la técnica quirúrgica (Rascovsky; Saadia).

Por ello, en el área de la investigación realizó siempre proyectos anátomo-quirúrgicos con la finalidad de proporcionar bases anatómicas útiles a la cirugía, abarcando muchos campos de la anatomía: cuello y tiroides, tórax, corazón, pulmones, mamas, estómago, duodeno, páncreas, vías biliares, colon y apéndice, sistema vascular, nervios simpáticos y bloqueos anestésicos, pared abdominal, perineo, entre otros. Esto le permitió describir nuevos procedimientos quirúrgicos que aplicó en la práctica asistencial con excelentes resultados.

Perfeccionó y elaboró mejoras en las disecciones y mostraciones de los preparados cadavéricos, con un método original "la bi-desinserción muscular", facilitando las prácticas de enseñanza y aprendizaje en los estudiantes de anatomía de la Carrera de Medicina (Martí; Régoli de Mullen).

Incentivó la investigación entre los colegas. Fue padrino y director de tesis doctorales de 32 médicos, cuyas temáticas se relacionaban con anatomía, embriología, clínica y técnica quirúrgica. Algunos de sus discípulos directos más destacados fueron Agustín Badano, Pedro Bianchi Donaire, Benjamín Javkin, Daniel Pastorino, Enrique Rascovsky y Adolfo Saadia (Albanese, 1961; Rascovsky; Lucas, 2005; Saadia; Régoli de Mullen).

Magister dixit. Algunas de las frases de Alfonso Albanese (recordando quizás un poco los aforismos de Hipócrates) dicen: "Interesarse en curar a un individuo, cobrando o sin cobrar, es una cosa de dioses. Quizás por eso la medicina estuvo asociada desde los tiempos más lejanos a la religión, y su desarrollo fue en algún momento coincidente". 
"En cuanto a las características que debe tener un cirujano, son: fuerza de carácter, autoridad, espíritu abierto al conocimiento, acción y riesgo, siempre con un gran sentido de equilibrio" (Régoli de Mullen).

Reconocimiento de colegas. Famosos cirujanos cardiovasculares que conocieron y frecuentaron al doctor Albanese, lo consideraron un maestro de la especialidad en Latinoamérica en las décadas de 1940 y 1950: René Favaloro (Buenos Aires, Argentina), Eduardo Palma (Montevideo, Uruguay), Mario Degni y Euryclides de Jesus Zerbini (San Pablo, Brasil), Rubens Carlos Mayall (Rio de Janeiro, Brasil), Arthur Mickelberg (Puerto Alegre, Brasil), Adolfo Escobar Pacheco (Santiago, Chile), Fernando Martorell Otzet (Barcelona, España), Joao Cid Dos Santos (Lisboa, Portugal), René Leriche y René Fontaine (Estrasburgo, Francia), entre otros; y también la médica clínica Helen Taussig (Baltimore, Estados Unidos de América) (Labra; Lucas, 1995; Lucas, 2005; Favaloro, 2008; Saadia; Régoli de Mullen).

Reconocimiento ítalo-argentino. Importantes personalidades de su región natal, lo visitaron en su casa hacia fines de 2003 brindándole homenajes como el premio a la "Trayectoria Meritoria" y lo declararon "Socio Honorario" de la Asociación Corigliano Calabria-Argentina y de la Asociación Sibaritas en el mundo.

Recordaron que Alfonso junto con su hermano Pascual, colaboraban en la "Asociación Buen Corazón" (en la zona del Abasto) donde atendían gratuitamente, como médicos recién recibidos, a los italianos que lo requerían (Benvenuto, 2008).

Reconocimiento universitario. Según el Rector de la Universidad del Salvador, Dr. Alejandro Tobías: "su ausencia impuso un gran vacío y el recuerdo siempre vivo del afecto. Fue, Albanese, un ejemplo de entrega a la vocación docente y a la investigación por la que transitó con fecundidad su vida. Con sus trabajos científicos enriqueció a nuestra Universidad y a la Medicina Argentina" (Régoli de Mullen).

El 4 de mayo de 2006, fecha en que el Maestro hubiese cumplido 100 años, las autoridades de la Facultad de Medicina de esa universidad dispusieron -en un conmovedor homenaje- que el Aula de la Cátedra de Anatomía en la que trabajó, lleve su nombre por sus méritos docentes, médicos y humanos (Régoli de Mullen).

El 23 de octubre de 2012, en el Aula Magna de esa Facultad se presentó un libro que reunió una serie de entrevistas que le habían hecho en su último año de vida, realizadas por la escritora Alicia Régoli de Mullen. Asistió el entonces Arzobispo de la ciudad de Buenos Aires, el Cardenal Jorge Mario Bergoglio, quien unos meses después sería designado Papa. Bergoglio y Albanese se conocían de la época cuando la Facultad funcionaba en el Colegio del Salvador y estaban ambas administradas por la Compañía de Jesús (Figs. 6 y 7).

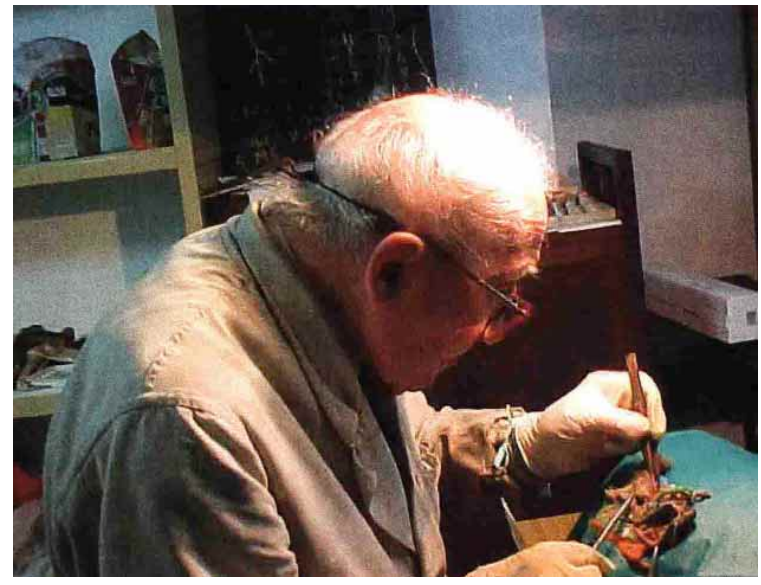

Fig. 6. El maestro Albanese disecando en la Cátedra de Anatomía.

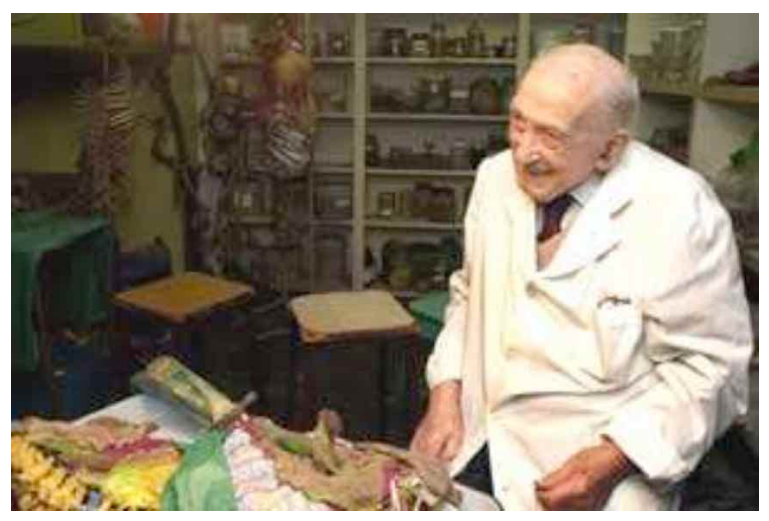

Fig. 7. El profesor Albanese en la Cátedra de Anatomía.

Reconocimiento societario. La Asociación Panamericana de Anatomía recordó la vida y obra del doctor Albanese, como uno de sus fundadores en una reunión científica: el XIV Simposio Ibero Latinoamericano de Terminología (SILAT), que se realizó en el Palacio de Medicina, en la ciudad de México, en julio de 2016, con motivo del $50^{\circ}$ aniversario de su fundación.

La personalidad. Su personalidad era apasionada, con una memoria prodigiosa. Creativo, inteligente y con muchos conocimientos generales. Sencillo, entusiasta, pícaro y expresivo. Afectivo, solidario y generoso. Escritor y dibujante excelente. Trabajador incansable. Una personalidad sorprendente con una historia de vida fascinante.

Su última cirugía la realizó a los 85 años en el "Sanatorio Anchorena" ayudado por unos de sus hijos, en una paciente con una hernia crural con anestesia local, con el mismo entusiasmo y destreza que siempre. 
CONCLUSIONES. La extensa e incansable tarea docente del doctor Albanese en universidades, en hospitales y en reuniones científicas ha contribuido a la formación de numerosas generaciones de médicos argentinos y de otros países.

Es un eslabón de singular jerarquía en la medicina argentina, tanto en la anatomía como en la cirugía, disciplinas que cultivó con pasión, dedicación y estudio. Creador de técnicas quirúrgicas que simplificaron las cirugías de su tiempo. Un verdadero artífice de la cirugía a partir de la anatomía y la embriología.

Un pionero de la cirugía cardiovascular en su país y en Latinoamérica, en las décadas de 1940 y 1950, en la que alcanzó un altísimo nivel, lo que lo ubicó también en un lugar destacado internacionalmente entre sus pares del mundo.

Su trayectoria sirve de modelo para los anatomistas y cirujanos de las actuales generaciones y las que han de venir.

\section{AGRADECIMIENTOS}

A los Profesores doctores Alfonso Miguel y Eduardo Francisco Albanese, hijos de Alfonso Roque Albanese, por las invalorables contribuciones a este artículo. Al maestro Alberto Devoto por la exhaustiva corrección de la redacción y los numerosos aportes brindados.

LOSARDO, R. J.; CRUZ, G. R.; PRATES, J. C.; RODRÍGUEZ, T. A.; PRATES, N. E. V. B.; ARTEAGA, M. M. \& CABRAL, R. H. Alfonso Roque Albanese: Latin American pioneer of heart surgery. Tribute from the Pan American Association of Anatomy. Int. J. Morphol., 35(3):10161025, 2017.

SUMMARY: Alfonso Roque Albanese (1906-2005) is considered a pioneer of Latin American heart surgery because of his important contributions. He was founding member of the Pan American Association of Anatomy. Researcher and teacher of anatomy and surgery, joining both disciplines.

KEY WORDS: Alfonso Roque Albanese; Pan American Association of Anatomy; Anatomy; Heart Surgery; Latin American.

\section{REFERENCIAS BILIOGRÁFICAS}

Albanese, A. R. Antecedentes, Títulos y Trabajos. Buenos Aires, Argentina, 1961.

Albanese, A. R. El apéndice, la Apendicitis y la Apendicectomía. Buenos Aires, Editorial López, 1977.

Apeceche, L. L. Eventración. Prensa Med. Argent., 67(6):196-8, 1980.

Benvenuto, A. Alfonso Rocco Albanese. Un Uomo Conosciuto per Caso. Corigliano, Editorial Aurora, 2008.

Bernardello, E. T. L.; Chacón, R.; Margossian, J. \& Mosto, A. Recientes avances en el diagnóstico y tratamiento del cáncer de la mama. Rev. Argent.
Cir., 1984(Supl.):57-142, 1984

Brana, R.; Chullmir, R.; Duarte, J.; Geragthy, F.; Cursi, A. \& Cervini, O. E. Maniobra de Wiart-Vautrin-Kocher. Prensa Med. Argent., 70(18):809-12, 1983.

Buroni, J. R. El apoyo sanitario que los argentinos brindaron a los franceses. Rev. Asoc. Med. Argent., 129(4):5-7, 2016.

Cano, C. A.; Yarade, M. E. \& Gutiérrez, N. Las incisiones de descarga de Albanese en el cierre de defectos catastróficos. Rev. Hispanoam. Hernia, 4(4):145-55, 2016

Christmann, F. E.; Ottolenghi, C. E.; Raffo, J. M. \& Von Golman, G. Técnica Quirúrgica. 11 ${ }^{\mathrm{a}}$ ed. Buenos Aires, Editorial El Ateneo, 1975.

Cruz Gutiérrez, R. \& Cruz Cruz, F. Desarrollo Normal y Anormal del Corazón (Anatomía, Histología y Embriología. Cardiopatías Congénitas). Ciudad de México, Editorial Trillas, 2012.

Devoto, A. A. Personalidades Argentinas. $N^{o} 1$. Raquel Navarro Viola, Arturo Illia, Enrique T. Susini. Buenos Aires, Argentina, 2016.

Favaloro, R. G. Recuerdos de un Médico Rural. Buenos Aires, Edición Debolsillo, 2008.

Figueroa, M. A. 80 Años de la Academia Argentina de Cirugía. Buenos Aires, Edición de la Asociación Argentina de Cirugía, 1993.

Finochietto, R. Cirugía Básica. Buenos Aires, Editorial López, 1962.

Gotta, H. C. \& Buzzi, A. E. Recuerdos Fotográficos de Nuestra Medicina. Tomo 1. Buenos Aires, Editorial Diagnóstico Médico, 2012.

Guerrino, A. A. Tríptico de la Medicina Argentina: Posadas-Agote-Castex. Prensa Med. Argent., 68(Supl.):25-30, 1981.

Labra, G. La Clínica Albanese. Archivos de la Sociedad de Cirujanos de Chile, 1956.

Losardo, R. J. Pan-American Association of Anatomy: Historic review and eminent rules. Int. J. Morphol., 27(4):1345-52, 2009.

Losardo, R. J.; Cruz Gutiérrez, R.; Prates, J. C.; Moscovici, M.; Rodríguez Torres, A. \& Arteaga Martínez, M. Sergey Fedoroff: A Pioneer of the neuronal regeneration. tribute from the Pan American Association of Anatomy. Int. J. Morphol., 33(2):794-800, 2015.

Losardo, R. J.; Binvignat Gutiérrez, O.; Cruz Gutiérrez, R. \& Aja Guardiola, S. La anatomía y las prácticas adivinatorias en las antiguas civilizaciones. Rev. Asoc. Med. Argent., 129(2):13-22, 2016.

Lucas, M. A. Anecdotario Cardiovascular. Vivencias Cordiales. Buenos Aires, Editorial Científica Graphos, 2005.

Lucas, M. A. Pioneros de la cirugía vascular: Dr. Alfonso Albanese, semilla y espejo. Actas Cardiovasc., 6(1):116-22, 1995.

Majul, E. J. Técnica de Albanese para la eventración post-apendicectomía con incisión de Rockey o Mc Burney. Prensa Med.Argent., 68(17):752-5, 1981.

Martí, M. L. Necrológica. Alfonso Roque Albanese (1906-2005). Prensa Med. Argent., 92(7):481-2, 2005.

Pastorino, D. E. Incisiones de descarga muscular de Albanese profilácticas. Prensa Med. Argent., 69(1):31-4, 1982.

Pataro, V. La técnica quirúrgica de Enrique Finochietto a través de Ricardo Finochietto. Prensa Med. Argent., 70(12):481-6, 1983.

Rascovsky, E. El Bálsamo en la Herida. Buenos Aires, Editorial La Lámpara, 2002.

Régoli de Mullen, A. Un Maestro de 99 Años. La Vida del Doctor Alfonso Roque Albanese. Buenos Aires, Ediciones Universidad del Salvador, 2011.

Saadia, A. Don Alfonso Roque Albanese: Maestro de los maestros. Rev. Argent. Cir. Cardiovasc., 7(3):154-9, 2009.

Vacarezza, O. A. 400 años de cirugía en Buenos Aires. Rev. Argent. Cir., 40(34):120-34, 1981.

Zancolli, E. A. Escuela quirúrgica Finochietto: un método de enseñar cirugía. Rev. Asoc. Med. Argent., 115(2):8-17, 2002.

Dirección para correspondencia:

Recibido : 26-04-2017

Prof. Dr. Ricardo Jorge Losardo

Aceptado: 28-06-2017

Lavalle 1844, 5to. "30". (1051)

Ciudad de Buenos Aires - ARGENTINA

E-mail: ricardo.losardo@usal.edu.ar 